BULLETIN OF THE

AMERICAN MATHEMATICAL SOCIETY

Volume 80, Number 1, January 1974

\title{
BIFURCATION AND STABILITY FOR A NONLINEAR PARABOLIC PARTIAL DIFFERENTIAL EQUATION
}

\author{
BY NATHANIEL CHAFEE AND E. F. INFANTE ${ }^{1}$
}

Communicated by Hans Weinberger, March 29, 1973

This note is a brief report on some research conducted by the authors in 1971. A complete report on this same research is scheduled to appear in a separate article [2].

Let $f$ be a given function continuously mapping the real line $R$ into itself. Let $\lambda$ be a given nonnegative real number. Let $\phi:[0, \pi] \rightarrow R$ be any $C^{1}$-smooth function such that $\phi(0)=\phi(\pi)=0$. We shall be discussing the following problem. Find a function $u$ continuously mapping the domain $\{(x, t): 0 \leqq x \leqq \pi, 0 \leqq t<+\infty\}$ into $R$ such that (i) the partial derivative $u_{\infty}$ is defined and continuous on $[0, \pi] \times[0,+\infty)$; (ii) the partial derivatives $u_{t}$ and $u_{x x}$ are defined and continuous on $[0, \pi] \times(0,+\infty)$; (iii) $u$ satisfies the equations

$\begin{array}{llll}\text { (1a) } & u_{t}(x, t)=u_{x x}(x, t)+\lambda f(u(x, t)) & & (0 \leqq x \leqq \pi, 0<t<+\infty) \\ \text { (1b) } & u(0, t)=u(\pi, t)=0 & & (0 \leqq t<+\infty) \\ \text { (1c) } & u(x, 0)=\phi(x) & & (0 \leqq x \leqq \pi) .\end{array}$

By a solution of (1) we mean a function $u$ having the properties just specified.

Our goal in studying (1) is to determine the asymptotic behavior of its solutions $u$ as $t \rightarrow+\infty$. We shall discuss this asymptotic behavior in terms of bifurcation and stability phenomena exhibited by (1).

Other authors have studied this same type of problem for parabolic partial differential equations. Specifically, we mention the works by Keller and Cohen [7] and by Sattinger [11], [12]. The basic tool in these papers is the maximum principle for parabolic and elliptic partial differential equations. We also mention a recent paper by Auchmuty [1] in which the fundamental approach is the use of Liapunov methods. Our own work [2] to be described here is also based on Liapunov methods.

In our investigation we assume that $f$ satisfies the following hypotheses:

$\left(\mathrm{H}_{1}\right) f$ is a $C^{2}$-smooth function mapping of $R$ into itself.

$\left(\mathbf{H}_{2}\right) f(0)=0$ and $f^{\prime}(0)>0$.

AMS (MOS) subject classifications (1970). Primary 35B40; Secondary 35B35, $35 \mathrm{~K} 35$.

1 This research was supported in part by the National Aeronautics and Space Administration under grant NGL 40-002-015, and in part by the U.S. Army Research grant DA-ARO-D-31-124-71-G12. 
$\left(\mathrm{H}_{3}\right) \lim \sup _{|\xi| \rightarrow+\infty} \xi^{-1} f(\xi) \leqq 0$.

$\left(\mathrm{H}_{4}\right) \operatorname{sgn} f^{\prime \prime}(\xi)=-\operatorname{sgn} \xi$ for all $\xi \in R$.

In that which follows we shall let $X$ denote the space of all $C^{1}$-smooth functions $\phi:[0, \pi] \rightarrow R$ such that $\phi(0)=\phi(\pi)=0$. On $X$ we impose a norm \|\|$_{1}$ by setting $\|\phi\|_{1}=\sup \left\{\left|\phi^{\prime}(x)\right|: 0 \leqq x \leqq \pi\right\}$ for all $\phi \in X$. $X$ is a Banach space under \|\|$_{1}$.

It can be shown that, for any $\phi \in X$ and $\lambda \in[0,+\infty)$, Equations (1) have a unique solution $u(\phi, \lambda)$ defined on $[0, \pi] \times[0,+\infty)$. For any $\phi \in X, \lambda \in[0,+\infty), x \in[0, \pi]$, and $t \in[0,+\infty)$, we can let $u(x, t ; \phi, \lambda)$ denote the value of $u(\phi, \lambda)$ at $(x, t)$. With this in mind, we can define, for any $\lambda \in[0,+\infty)$, a nonlinear semigroup $\left\{U_{\lambda}(t)\right\}$ on $X$ by setting $U_{\lambda}(t) \phi=u(\cdot, t ; \phi, \lambda)$ for all $\phi \in X$ and $t \in[0,+\infty)$. It can be shown that $\left\{U_{\lambda}(t)\right\}$ is strongly continuous on $X$.

Let $\lambda \in[0,+\infty)$. By an equilibrium solution of (1) (corresponding to $\lambda$ ) we mean a function $u_{0} \in X$ such that $U_{\lambda}(t) u_{0}=u_{0}$ for all $t \in[0,+\infty)$. By virtue of $\left(\mathrm{H}_{2}\right)$, the origin $\phi_{0}=0$ in $X$ is an equilibrium solution of (1) for every $\lambda \in[0,+\infty)$.

To discuss the existence of other equilibrium solutions for (1) we introduce the linearized steady-state problem

$$
\begin{array}{ll}
v^{\prime \prime}(x)+\lambda f^{\prime}(0) v(x)=0 & (0 \leqq x \leqq \pi) \\
v(0)=v(\pi)=0 &
\end{array}
$$

Taking into account $\left(\mathrm{H}_{2}\right)$, we see that (2) has eigenvalues $\lambda_{n}={ }^{\text {def }} n^{2} / f^{\prime}(0)$, $n \geqq 1$, and that $0<\lambda_{1}<\lambda_{2}<\cdots<\lambda_{n}<\cdots$. We are now ready to state our first theorem.

THEOREM 1. For any integer $n \geqq 1$ and any number $\lambda \in\left(\lambda_{n},+\infty\right)$, Equations (1) have a unique pair of equilibrium solutions $u_{n}^{ \pm}(\lambda)$ such that (i) $u_{n}^{ \pm}(\lambda)$ has $n+1$ simple zeros $x_{0}^{ \pm}(\lambda), x_{1}^{ \pm}(\lambda), \cdots, x_{n}^{ \pm}(\lambda)$ in $[0, \pi]$ with

$$
0=x_{0}^{ \pm}(\lambda)<x_{1}^{ \pm}(\lambda)<\cdots<x_{n}^{ \pm}(\lambda)=\pi ;
$$

(ii) $(-1)^{q} u_{n}^{ \pm}(x ; \lambda) \gtrless 0$ for each integer $q=0,1, \cdots, n-1$ and each $x \in$ $\left(x_{n}^{ \pm}(\lambda), x_{q+1}^{ \pm}(\lambda)\right)$. Furthermore, the mapping $\lambda \mapsto u_{n}^{ \pm}(\lambda)$ from $\left(\lambda_{n},+\infty\right)$ into $X$ is continuous on $\left(\lambda_{n},+\infty\right)$, and $u_{n}^{ \pm}(\lambda) \rightarrow 0$ as $\lambda \rightarrow \lambda_{n}$, and $\left\|u_{n}^{ \pm}(\lambda)\right\|_{1} \rightarrow+\infty$ as $\lambda \rightarrow+\infty$. Finally, for any $\lambda \in[0,+\infty)$, Equations (1) have no equilibrium solutions other than the zero solution $u_{0}=0$ and those elements $u_{n}^{ \pm}(\lambda), n \geqq 1$, such that $\lambda_{n} \leqq \lambda$.

The assertion $u_{n}^{ \pm}(\lambda) \rightarrow 0$ as $\lambda \rightarrow \lambda_{n}$ has the obvious geometric interpretation that the pair $u_{n}^{ \pm}(\lambda)$ bifurcates from the origin $u_{0}=0$ as $\lambda$ increases from $\lambda_{n}$.

The existence of $u_{n}^{ \pm}(\lambda)$ for Equations (1) can be easily deduced from a general result due to Crandall and Rabinowitz [3, p. 1084]. However, that same result does not guarantee the uniqueness of $u_{n}^{ \pm}(\lambda)$ stated in 
Theorem 1. Our proof in [2] for Theorem 1 is based on phase-energy methods.

Now we state another theorem.

THeOREM 2. For any $\phi \in X$ and any $\lambda \in[0,+\infty)$, there exists an equilibrium solution $u_{0}(\phi, \lambda)$ of (1) such that $U_{\lambda}(t) \phi \rightarrow u_{0}(\phi, \lambda)$ as $t \rightarrow+\infty$.

The question arises, given $\phi \in X$ and $\lambda \in[0,+\infty)$, to which of the equilibrium solutions described in Theorem 1 is $u_{0}(\phi, \lambda)$ equal? A partial answer to this query is given in the following theorem.

THeORem 3. For any $\lambda \in\left[0, \lambda_{1}\right]$, the zero solution $u_{0}=0$ of $(1)$ is globally asymptotically stable in the sense of Liapunov. In particular, for each $\phi \in X$ and $\lambda \in\left[0, \lambda_{1}\right]$, we have $\left\|U_{\lambda}(t) \phi\right\|_{1} \rightarrow 0$ as $t \rightarrow+\infty$. For any $\lambda \in\left(\lambda_{1},+\infty\right)$, the zero solution $u_{0}=0$ of (1) is unstable. For any $\lambda \in\left[\lambda_{1},+\infty\right)$, the solutions $u_{1}^{ \pm}(\lambda)$ are each asymptotically stable in the sense of Liapunov. Finally, for any integer $n \geqq 2$ and any $\lambda \in\left[\lambda_{n},+\infty\right)$, the solutions $u_{n}^{ \pm}(\lambda)$ are each unstable.

We remark that the stability properties of $u_{1}^{ \pm}(\lambda)$ and $u_{0}=0$ asserted in Theorem 3 can be obtained using methods developed by Sattinger (see [11, pp. 992-993]).

Our proofs for Theorems 1-3 appear in [2]. Now we shall briefly sketch these proofs.

Our approach to studying Equations (1) is to interpret (1) as a dynamical system on $X$ and then to apply certain methods associated with the Liapunov theory of stability. The methods we have in mind are set forth in [4], [5] and [9] and are often referred to as the invariance principle in stability theory.

An essential tool in our use of the invariance principle is the following Liapunov functional:

(3) $V_{\lambda}(\phi)=\int_{0}^{\pi}\left\{\frac{1}{2} \phi^{\prime}(x)^{2}-\lambda \int_{0}^{\varphi(x)} f(\xi) d \xi\right\} d x \quad(\phi \in X, \lambda \in[0,+\infty))$.

For each $\lambda \in[0,+\infty)$, Equation (3) defines a functional $V_{\lambda}$ mapping $X$ into $R$. For any $\phi \in X$ and $\lambda \in[0,+\infty)$, it can be shown that

$$
\dot{V}_{\lambda}\left(U_{\lambda}(t) \phi\right)=-\int_{0}^{\pi}\left|u_{t}(x, t ; \phi, \lambda)\right|^{2} d x \quad(t>0) .
$$

Consider any $\phi \in X$ and $\lambda \in[0,+\infty)$. Using $V$, one can show that $u(\phi, \lambda)$ has a nonempty compact connected invariant $\omega$-limit set $\omega(\phi, \lambda) \subset$ $X$. Here, one also uses the invariance principle referred to two paragraphs above. That same principle together with Equation (4) tells us that any element in $\omega(\phi, \lambda)$ must be an equilibrium solution of (1). 
Therefore, one now seeks the equilibrium solutions of Equations (1). This leads us to study the two-point boundary-value problem

$$
\begin{aligned}
& u^{\prime \prime}(x)+\lambda f(u(x))=0 \quad(0 \leqq x \leqq \pi, 0 \leqq \lambda<+\infty) \\
& u(0)=u(\pi)=0 .
\end{aligned}
$$

Our results concerning (5) are stated in Theorem 1. In particular, we see that, for any $\lambda \in[0,+\infty)$, each equilibrium solution of (1) is isolated in $X$. Hence, for any $\phi \in X$ and $\lambda \in[0,+\infty)$, the set $\omega(\phi, \lambda)$ consists of exactly one equilibrium solution of (1). From this there follows Theorem 2.

Theorem 3 is established using arguments from the classical theory of calculus of variations. We shall not attempt to describe these arguments here.

\section{REFERENCES}

1. J. F. G. Auchmuty, Lyapunov methods and equations of parabolic type, Proc. Battelle Summer Inst. Applications of Non-Linear Analysis, Lecture Notes in Math., Springer-Verlag, Berlin and New York, 1972. (to appear).

2. N. Chafee and E. F. Infante, A bifurcation problem for a nonlinear partial differential equation of parabolic type, Applicable Anal. (to appear).

3. M. G. Crandall and P. H. Rabinowitz, Nonlinear Sturm-Liouville eigenvalue problems and topological degree, J. Math. Mech. 19 (1969-70), 1083-1102. MR 41 \#3874.

4. J. K. Hale and E. F. Infante, Extended dynamical systems and stability theory, Proc. Nat. Acad. Sci. U.S.A. 58 (1967), 405-409. MR 36 \#1769.

5. J. K. Hale, Dynamical systems and stability, J. Math. Anal. Appl. 26 (1969), 39-59. MR 39 \#5896.

6. Ja. I. Kanel', Stabilization of solutions of the Cauchy problem for equations encountered in combustion theory, Mat. Sb. 59 (101) (1962), suppl., 245-288. (Russian) MR 28 \#367.

7. H. B. Keller and D. S. Cohen, Some positone problems suggested by nonlinear heat generation, J. Math. Mech. 16 (1967), 1361-1376. MR 35 \#4552.

8. A. Kolmogoroff, I. Petrovsky and N. Piscounoff, Etude de l'équation de la diffusion avec croissance de la quantité de matière et son application à un problème biologique, Bulletin de l'Université d'Etat à Moscou, Serie Internationale, Vol. I, 1937.

9. J. P. LaSalle, An invariance principle in the theory of stability, Differential Equations and Dynamical Systems (Proc. Internat, Sympos., Mayaguez, P.R., 1965), Academic Press, New York, 1967, pp. 277-286. MR 37 \#1722.

10. B. J. Matkowsky, A simple nonlinear dynamic stability problem, Bull. Amer. Math. Soc. 76 (1970), 620-625. MR 41 \#2194.

11. D. H. Sattinger, Monotone methods in nonlinear elliptic and parabolic boundary value problems, Indiana Univ. Math. J. 21, (1972), 979-1000.

12. ——, Topics in stability and bifurcation theory, Springer-Verlag, New York, 1973, Chapter II.

Division of Applied Mathematics, Brown University, Providence, Rhode IsLAND 02912. (Current address of E. F. Infante.)

Current address (Nathaniel Chaffee): School of Mathematics, Georgia Institute of Technology, Atlanta, Georgia 30332 